OPEN ACCESS

Edited by:

Phil Salmon,

Bruker-microCT, Belgium

Reviewed by:

Paul Zaslansky,

Charité Hospital, Germany

Kostas Verdelis,

University of Pittsburgh, USA

*Correspondence:

David M. L. Cooper,

Department of Anatomy and Cell Biology, University of Saskatchewan,

107 Wiggins Road, Saskatoon, SK S7N 5E5, Canada dml.cooper@usask.ca

Specialty section:

This article was submitted to Bone Research, a section of the journal

Frontiers in Endocrinology

Received: 03 February 2015

Accepted: 24 July 2015

Published: 11 August 2015

Citation:

Harrison KD and Cooper DML (2015) Modalities for visualization of cortical bone remodeling: the past, present, and future.

Front. Endocrinol. 6:122. doi: 10.3389/fendo.2015.00122

\section{Modalities for visualization of cortical bone remodeling: the past, present, and future}

\author{
Kimberly D. Harrison and David M. L. Cooper* \\ Department of Anatomy and Cell Biology, University of Saskatchewan, Saskatoon, SK, Canada
}

Bone's ability to respond to load-related phenomena and repair microdamage is achieved through the remodeling process, which renews bone by activating groups of cells known as basic multicellular units (BMUs). The products of BMUs, secondary osteons, have been extensively studied via classic two-dimensional techniques, which have provided a wealth of information on how histomorphology relates to skeletal structure and function. Remodeling is critical in maintaining healthy bone tissue; however, in osteoporotic bone, imbalanced resorption results in increased bone fragility and fracture. With increasing life expectancy, such degenerative bone diseases are a growing concern. The three-dimensional (3D) morphology of BMUs and their correlation to function, however, are not well-characterized and little is known about the specific mechanisms that initiate and regulate their activity within cortical bone. We believe a key limitation has been the lack of 3D information about BMU morphology and activity. Thus, this paper reviews methodologies for 3D investigation of cortical bone remodeling and, specifically, structures associated with BMU activity (resorption spaces) and the structures they create (secondary osteons), spanning from histology to modern ex vivo imaging modalities, culminating with the growing potential of in vivo imaging. This collection of papers focuses on the theme of "putting the 'why' back into bone architecture." Remodeling is one of two mechanisms "how" bone structure is dynamically modified and thus an improved 3D understanding of this fundamental process is crucial to ultimately understanding the "why."

Keywords: basic multicellular unit, bone, remodeling, micro-CT, synchrotron

\section{Introduction}

Bone tissue is three-dimensionally (3D) complex in structure and undergoes continual dynamic change. Despite its rigid structure, it is remarkable in its ability to adapt in response to mechanical stimuli associated with loading and to microdamage endured throughout life. Since Clopton Havers' (1) description of "Haversian" canals and iconic works describing microscopic bone structure/function relationships $(2,3)$, it has been well appreciated that bone renews itself via the turnover of tissue, which we have come to know as "remodeling" (4). Remodeling is critical for maintaining healthy bone tissue; however, it can also lead to age-related bone loss through an imbalance between osteoclastic (bone resorption) and osteoblastic (bone formation) activity. A progressive deficit in bone formation leads to enlarged osteonal canals and thus increased cortical porosity (5-7). Ultimately, this contributes to bone's fragility which is characteristic of osteoporosis $(7,8)$. Related fractures are significant events in the lives of those afflicted and are frequently associated with serious complications and even 
mortality. With increasing life expectancy, osteoporosis and other degenerative diseases of bone are a growing concern for health care systems worldwide (9). As such, study of the spatio-temporal regulation of remodeling is a topic of great significance within bone biology with the potential to impact many lives.

First described by Frost (10), basic multicellular units (BMUs) are the cellular groups responsible for carrying out the remodeling process. In cortical bone, this is achieved through the localized resorption of a cylindrical space (osteoclastic "cutting cone") followed by concentric infilling of new tissue (osteoblastic "closing cone") (Figure 1). The resulting structure is referred to as a secondary osteon (synonymous with "Haversian system"). As BMUs organizationally lie between the level of the cell and that of the tissue, Frost referred to them as "intermediary" (11). Despite decades of study, our understanding of the intermediary organization of bone remains rudimentary. BMUs are temporary collections of cells brought together to turnover a discrete packet of bone. Their course through bone tissue, their "behavior", is challenging to directly probe, and thus much of our understanding has been inferred from osteon morphology. The orientations of secondary osteons appear to reflect principal stresses (12-16), and thus it has been hypothesized that the progression of BMUs is influenced by mechanical stimuli. This is not surprising as the

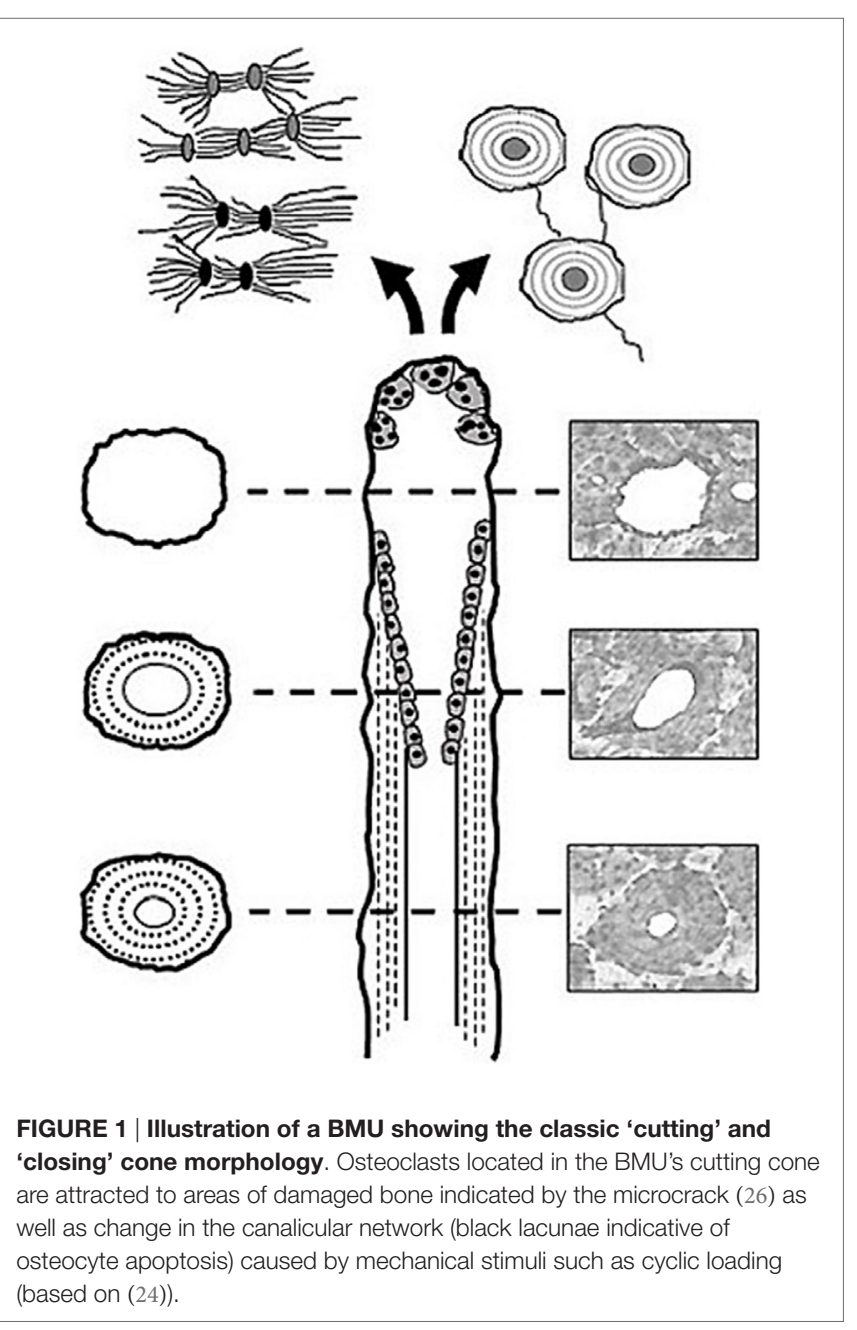

two-dimensional (2D) geometry of secondary osteons has been linked to the function of the bones in which they are found (17, 18) and resultant mechanical strains (19). Additional examples include intra-element regional (i.e., anterior, posterior, lateral, medial) variation in osteon morphology $(20,21)$ and a relation between osteon size and weight observed in humans (22). To explain the link between mechanics and BMU orientation, computational (in silico) modeling has looked to stimuli such as localized strain (23) and strain-related fluid flow (24) around cutting cones. Such in silico models continue to become increasingly sophisticated, extending into the realm of simulation (25). All models, however, have relied upon highly idealized BMU morphology, and it is unclear how compatible their findings are with the more complex $3 \mathrm{D}$ morphologies which have been reported.

Another hypothesis regarding BMU regulation holds that their activities are spatially "targeted" (27) to remove damage manifested as microcracks $(16,28-30)$. While debate remains over the degree to which remodeling is targeted vs. untargeted (26), if targeting occurs, even for a portion of remodeling events, there must be mechanisms which actively steer BMUs toward damaged areas (Figure 1). This has been envisioned as attraction toward an "effective damage removal area," which provides the means by which the osteoclasts of a BMU are drawn toward microdamage (15). Although it has been demonstrated that remodeling-related resorption spaces are associated with microcracks (31), active steering has yet to be empirically demonstrated. It is possible that BMUs are simply initiated in damaged areas. Further, other stimuli clearly play a role, and thus a clear dichotomy between targeted vs. non-targeted views of BMU regulation is problematic and they need not necessarily be mutually exclusive (27). The classical view of remodeling has always been envisioned as a multi-functional role - including mechanical and physiological functions (26) such as calcium homeostasis. Efforts to disentangle the multi-faceted regulation of remodeling would be greatly aided by more and better $3 \mathrm{D}$ data regarding BMUs and related structures.

In sum, the capacity to directly test hypotheses related to regulation of BMU activity and/or the validation of in silico models is limited by a general lack of 3D data. Indeed, the activity of BMUs has largely been inferred from 2D observation of the secondary osteons they create. Our appreciation of the 3D structure of secondary osteons is similarly limited, and those data which are available (discussed below) consistently hint at greater structural complexity than commonly appreciated. Improving our 3D understanding of cortical bone microarchitecture would, thus, enhance our understanding of the remodeling process. As such, the objective of this paper is to provide an overview of methodologies for 3D investigation of cortical bone remodeling and, specifically, structures associated with BMU activity (resorption spaces) and the structures they create (secondary osteons). This review will survey a range of approaches spanning from histology to modern exvivo imaging modalities, culminating with the growing potential of in vivo imaging. As such, it will span past, present, and emerging approaches. This collection of papers focuses on the theme of "putting the " why" back into bone architecture." Remodeling is one of two mechanisms "how" bone structure is dynamically modified, 
and thus an improved 3D understanding of this fundamental process is crucial to ultimately understanding the "why."

\section{Past: Histological Approaches}

Long before the advent of modern 3D imaging modalities [e.g., confocal microscopy, scanning electron microscopy, and microcomputed tomography (micro-CT)], traditional light microscopy yielded a wealth of information about bone microarchitecture. Early applications of light microscopy revealed remodelingrelated structures including resorption spaces, mature osteons, and the canals within these osteons. The study of ground sections led to the first hypotheses pertaining to functional significance. Among the earliest of observations was a link between the extent of remodeling and age. Amprino and Bairati's (32) study of compact bone from humans and animals was one of the first to show an association between age and osteon population density (i.e. the number of osteons $/ \mathrm{mm}^{2}$ ). Why this occurs, either through targeted replacement or through the accumulation of stochastic events, remains a central debate in the field. It is not surprising then than much remains to be learned from histology and that histology, in various forms, remains a mainstay of bone biology to this day. There are, however, limitations to extrapolating $3 \mathrm{D}$ structure from sections - a leap which requires idealization and assumption (33). Indeed, histomorphology can vary significantly due to differences in sampling location. The formation of an osteon by a BMU is an excellent example. Remodeling can create a new osteon anywhere along a bone's diaphysis and the resorption space in which it forms can extend over several millimeters (34). Stout et al. (35) found that sectioning osteons at different points along their lengths could result in the erroneous classification of different morphological "types." They provided the example of a "dumbbell-shaped" osteon being explained as the product of sectioning through a branching event. Beyond morphology of individual osteons, the rate of remodeling can vary within a bone. Skedros et al. (18), for example, demonstrated that the number of osteons present in the forelimb bones of Rocky Mountain mule deer increased in a proximal to distal fashion. While larger fields of view (FOV) have become increasingly feasible through the use of tiling microscopes, FOV has been a significant limitation of microscopy. This is especially problematic for the measure of rate of remodeling - reflected by the measure "activation frequency," which is defined as the number of new BMUs present in a particular unit of bone (10). Activation frequency can directly affect overall bone mass, because "remodeling space" temporarily reduces overall bone mass until it is subsequently filled in Ref. (36). Thus, a high remodeling rate can, in part, account for low mass. Activation frequency can be measured in either $2 \mathrm{D}$ sections (BMUs/ $\mathrm{mm}^{2} /$ unit time) (10) or 3D volumes (BMUs/ $\mathrm{mm}^{3} /$ unit time) based on the number of BMUs created per unit volume of cortex per unit time (37). The 3D extent of BMUs - their length or "range" - is a very difficult parameter to assess from histology, even from longitudinal sections. This parameter has the potential to impact the regional assessment of activation frequency with longer resorption spaces having increased potential to be detected in section. Cooper et al. (34) micro-CT analysis of $99 \mathrm{BMUs}$ in human bone provided a measure of range varying between $\sim 0.8$ and $5.4 \mathrm{~mm}$ and most likely longer as they were limited to a $7 \mathrm{~mm}$ FOV and some remodeling events extended beyond. Keeping in mind the significant impact of sample site selection, activation frequency measures based on how many BMU-related resorption spaces are visible in sections acquired only millimeters apart from one another could be very different. Another problem with typical activation frequency measures is that, in section it can be difficult to differentiate BMU-related resorption spaces from other structures such as Volkmann's canals (36). That being said histology-based analytical techniques are still the gold standard for visualization of bone microstructure. Indeed, histology remains the primary means of investigating the relation between remodeling and microdamage. When identifying microdamage, in the form of microcracks, it is critical to ensure one is detecting in vivo damage and not artifacts of processing - particularly when utilizing grinding-based approaches as they can produce artifacts within bone that resemble cracks induced in vivo (38, 39). Microcracks are generally observed in $2 \mathrm{D}$ sections $(40,41)$, and thus their overall morphology, and relation to remodeling events, can be difficult to ascertain. For example, comparison of microcrack morphological features, such as size and shape, viewed in $2 \mathrm{D}$ has been shown to exhibit differences, to the extent that individual cracks look like entirely distinct structures (41, 42). This was seen to be the case in a study by Voide et al. (43), which found certain microcracks appeared linear and confined in the cross-sectional plane, whereas viewed in the perpendicular plane, they appeared diffuse.

Serial sectioning can alleviate some of these issues associated with 2D histology. Multiple sections increase the amount of bone analyzed and provide direct insight into the 3D nature of microarchitecture. Cohen and Harris' (44) analysis of serial decalcified sections from canine femora reported that osteons follow a spiral orientation around both the axis of the bone and axes of the osteons themselves. This seminal 3D study also reported that morphological characteristics vary in their distribution and this variation is linked to the specific locations within the bone. They observed that osteon cross-sectional area increased as they coursed distally throughout the bone and were also greater in the endosteal as opposed to subperisosteal regions (44), a finding suggestive of a mechanical influence on osteon morphology and thus BMU activity. Tappen's (45) work with block section staining using silver nitrate revealed morphological characteristics of osteonal canals, lacunae, osteocytes, and canaliculi. Tappen (45) highlighted numerous features of osteons, and the remodeling process which included levels of stain uptake in bone can be used as an indicator of mineralization; BMU-related resorption spaces are continuous with canals; these spaces can tunnel in opposite directions from one another or in only one direction, and some unforeseen force(s) dictate osteon/resorption space diameter as evidenced by the areas of mineralized bone resorbed in active areas of remodeling.

Despite its advantages, serial sectioning is challenging and thus only a few significant attempts to investigate cortical bone microstructure by this approach have been published $(35,44-46)$. Notably, these few studies have not found consensus with respect to some general aspects of osteon morphology. For example, Stout et al. (35) digital analysis of Tappen's (45) serial sections did not 
find evidence that osteons follow a spiral pattern around the axes of bone as suggested by Cohen and Harris (44). Rather, they found a complex pattern of branching osteons. Indeed, all 3D studies have revealed greater structural complexity than is frequently appreciated in the literature. While the literature is dominated by the "classical" BMU morphology presented in Figure 1, it should be noted that data indicating a complex diversity of remodeling-event types was reported in the mid 1960s by Johnson (4). We would argue that a lack of practical and efficient $3 \mathrm{D}$ techniques has contributed to the corresponding lack of information regarding BMU and osteon morphology. Beyond the difficult and tedious nature of existing serial-sectioning approaches, no small measure of luck is required to target these techniques to study individual BMUs and/or their related structures. Methodological difficulties ultimately led to the exploration of imaging-based approaches to the study of bone remodeling.

\section{Present: Ex Vivo Imaging}

Since its introduction by Feldkamp et al. (47), X-ray micro-CT has become the "gold standard" for the non-destructive 3D analysis of trabecular bone morphology. More recently, this technology has increasingly been applied to cortical bone to analyze the porous network of canals in $3 \mathrm{D}(6,14,34,48-53)$. With respect to the study of remodeling, micro-CT represents an excellent approach for detection of BMU-related resorption spaces since their distinctive cutting cones generally stand out from the relatively smaller canals of completed osteons. Another key advantage of micro-CT is its ability to non-destructively survey a large volume of bone for active remodeling events. An excellent example of this is our group's recent use of a laboratory micro-CT system (SkyScan 1172, Bruker, Belgium) to locate and trace BMUs in complete diaphyses of Ursus americanus (black bear) metacarpals and metatarsals without the requirement of sectioning (Figure 2) (54). In a more general sense, micro-CT can provide insight into remodeling through assessment of canal (and hence osteon) orientation (49) as well as the overall complexity of the canal network, which has been hypothesized to increase with cumulative remodeling activity $(34,48)$. The potential exists to examine osteon "steering" through assessment of localized canal orientation. Thus, the capabilities of micro-CT can alleviate or, at minimum, limit many issues associated with histological techniques for cortical bone: (1) interpolation of 3D structure from $2 \mathrm{D}$ sections is not needed as structures are observed directly and measured in 3D; (2) measures can be calculated in units of volume as opposed to area; (3) sample preparation is limited, eliminating structural alterations; (4) sample site location is less of an issue as visualization and analysis of larger volumes of interest - even entire bones - become feasible. These advantages are just beginning to be brought to bear on the ex vivo study of cortical remodeling, and micro-CT clearly has great potential to provide new insights through improved measures of morphology (including orientation, possible "steering," and 3D range) and activation frequency.

Despite its many benefits, micro-CT has several limitations. For cortical bone, the most notable is that while some delineation of osteon borders is possible from laboratory systems (55), this technology remains largely limited to detecting porosity at the vascular level and larger (e.g., osteonal canals and resorption

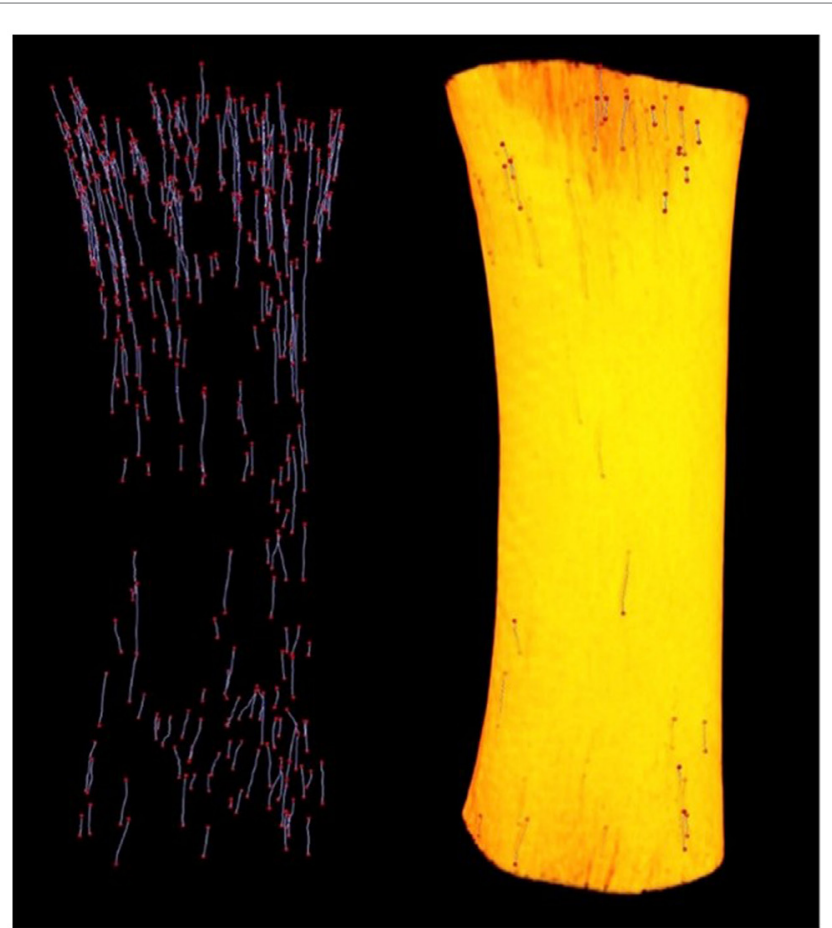

FIGURE 2 | Left: reconstructed micro-CT image of landmarked BMUs in a black bear metacarpal; right: 3D render of bone diaphysis superimposed over BMUs. Diaphysis length $=31.84 \mathrm{~mm}$.

spaces). Synchrotron radiation (SR) micro-CT, with its many advantages conveyed by greater X-ray flux, its monochromatic coherence, and high brilliance as a result of a small source size can be utilized to visualize smaller-scale structures including osteon borders $(56,57)$ and different osteon morphologies $(57)$, osteocyte lacunae (58-60) (Figure 3), and even microcracks (42, 43, 61, 62).

A limitation shared by both micro-CT and SR micro-CT is that, in general terms, higher resolution comes at the cost of field of view. A further related limitation is that as resolution increases, so does the radiation dose. Improving resolution by a factor of 2 requires a dose increase by a factor of 16 to maintain image quality in micro-CT (63). This relation provides the practical limit on the resolution for in vivo $\mathrm{X}$-ray based computed tomography. Thus, despite the fact that micro-CT imaging of trabecular bone microstructure in living animals is now commonplace (10-20 um voxel size), its application to the internal microstructure of cortical bone is not. An exception to this is the increasing use of high resolution-peripheral computed tomography (HR-pQCT) to analyze cortical porosity in humans, which will be discussed in the next section. The limited applications of micro-CT to the internal microstructure of cortical bone (e.g., osteonal canals) in animal models have all been ex vivo $(64,65)$. Working ex vivo, it is even possible to image microcracks utilizing barium sulfate as a contrast agent (66-68). With the vascular porosity of cortical bone beyond the reach of conventional in vivo micro-CT, it goes without saying that the resolutions required for microcrack imaging are not compatible with live animal imaging. Voide et al. (43) used SR micro-CT with a nominal 


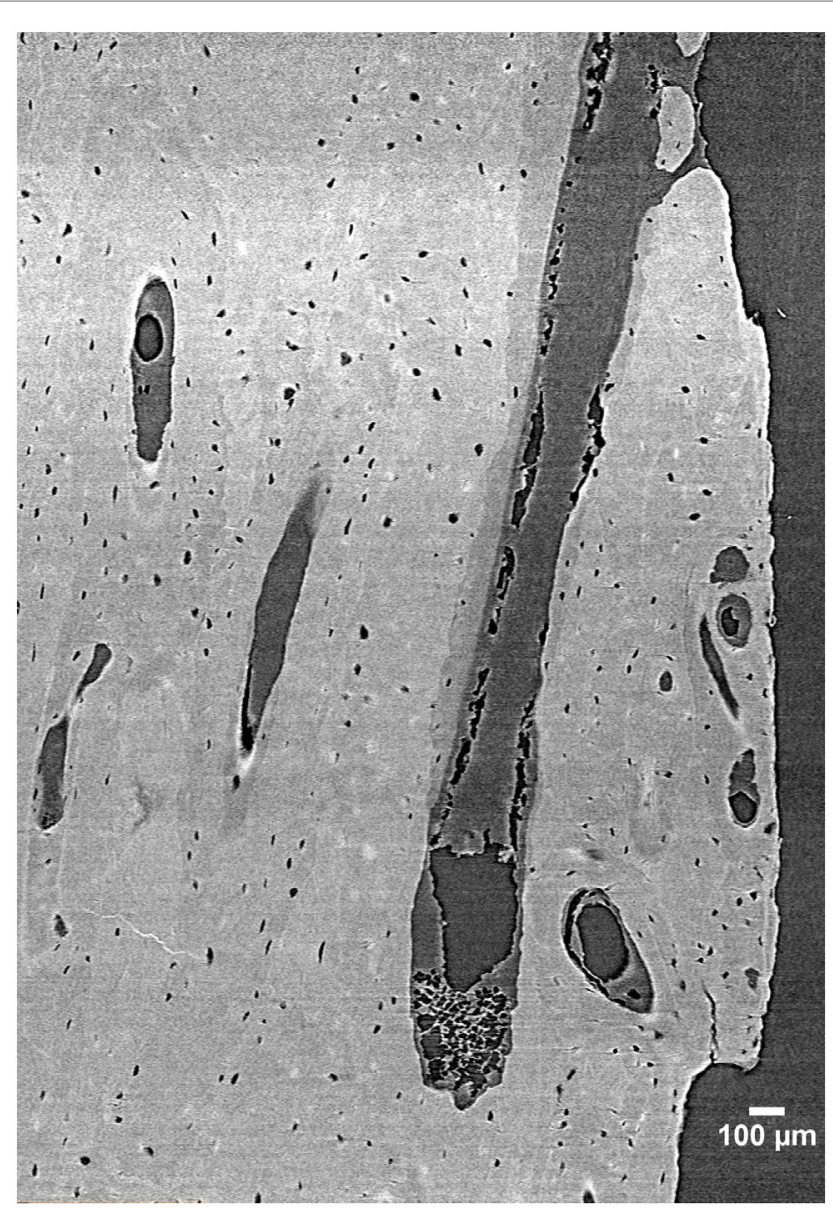

FIGURE 3 3 3D reconstruction of a human femur section depicting BMUs and osteocyte lacunae acquired by SR micro-CT at a $1.47 \mu \mathrm{m}$ resolution (58). Image provided by Dr. Yasmin Carter.

resolution of $700 \mathrm{~nm}$ to visualize microcracks, ex vivo, in the femora of mice. Even if radiation dose was not an issue, holding a living animal still for sub-micron imaging would present a significant challenge in and of itself. Thus, while much can be learned about remodeling through ex vivo 3D imaging, in vivo detection and tracking of remodeling-related structures would represent a significant step forward with still greater potential to improve our understanding of this process. As will be discussed, there are specific advantages of synchrotron-based imaging which have created the potential for in vivo imaging of cortical porosity - opportunities that are just beginning to be explored and thus lie on the future horizon.

\section{Future: In vivo Imaging}

A new generation of clinical research HR-pQCT scanners have given rise to a new opportunity for $3 \mathrm{D}$, in vivo, characterization of human bone, both trabecular and cortical. With an isotropic voxel size of $82 \mu \mathrm{m}, \mathrm{HR}-\mathrm{pQCT}$ has been at the forefront for microarchitectural analysis of the human appendicular skeleton (i.e., distal radius and tibia) $(69,71)$, as it is particularly advantageous for measuring cortical porosity (70). While HR-pQCT has been instrumental in studies concerned with osteoporosis induced change in microstructural cortical bone (71), its viability as a tool for targeting and tracking specific cortical structures, such as individual BMUs, is unclear due to resolution and other limitations associated with movement artifacts and the restriction to primary trabecular bone sites (i.e., wrists and ankles) (72). When one considers that the size of a single HR-pQCT voxel is on the same scale as the average canal diameter within human cortical bone, $\sim 120 \mu \mathrm{m}$ for pooled sexes (6), it becomes clear that this technology cannot resolve all osteonal canals. A recent study comparing HR-pQCT as a tool for measuring pore sizes against SR micro-CT showed that the accuracy of HR-pQCT was a factor of the size of the pores measured (70) - current resolution limits its ability to recognize small pores, thus introducing partial volume effects into analyses (72). That said, as BMU-related resorption spaces average $250 \mu \mathrm{m}$ in diameter [e.g., the average diameter of an osteon in humans (22)], HR-pQCT may indeed hold the potential of tracking individual remodeling events in human cortical bone. Thus, while HR-pQCT represents a powerful new tool for the assessment of cortical porosity, it is limited to the largest of cortical pores located in the ultra-distal peripheral skeleton.

Human-based studies are clearly an important avenue for study, but more mechanistic studies will require controlled model systems. When considering animal models - particularly smaller animals - the punishing relation between resolution and radiation dose complicates in vivo imaging. The risks associated with high $\mathrm{X}$-ray doses are not only harmful to a living subject in an acute sense, they include the potential to alter bone structure, leading to osteopenia, growth arrest, fracture, and malignancy (73) through altered osteoclast and osteoblast activity (74). For example, at a voxel size of $10.5 \mu \mathrm{m}$ and a radiation dose of $845.9 \mathrm{mGy}$, longitudinal scans of the hind limb in mice acquired over a 5-week period resulted in decreased bone volume and increased trabecular separation (75). Even though SR micro-CT makes in vivo imaging of cortical porosity more plausible through higher resolution and shorter scan times, the limitations imposed by radiation dose are still a factor. As will be discussed, the monochromatic (single X-ray energy) capabilities of synchrotrons enable alternative methods of developing contrast beyond absorption. These so-called "phase contrast" techniques are opening the door to new possibilities for in vivo imaging.

Several forms of phase contrast imaging, including in-line phase contrast, diffraction-enhanced imaging, and interferometer-based imaging, have been increasingly explored for their potential utilization in biomedical applications. An excellent overview of these methodologies is provided by Zhou and Brahme (76). Unlike conventional radiography, where images are based on the differences in X-ray absorption related to an object's internal structure, phase contrast images contain information related to the refractive index of an object because the images produced are derived from the pattern of interference created from diffracted and undiffracted waves (77). Differences in refractive index of the sample's internal structure will refract or bend the X-ray wavefront as they pass through the target and these differences can be used to generate contrast (76). For in-line phase contrast imaging - the simplest of these techniques to implement - detection of changes in X-ray 
refractive indices within a sample can be optimized by altering the distance between the object and the detector $(76,78)$ (Figure 4). Simple implementation and enhanced detection of internal features have made the fusion of SR computed tomography and phase contrast imaging particularly advantageous for visualization of microscopic bone structures such as osteocyte lacunae $(58,79$, 80 ), and even nanoscopic structures such as the lacuno-canalicular network (59). Phase contrast is not dependent on X-ray attenuation and thus higher energies, where attenuation is lower, can be employed (76). This creates the possibility of higher resolution with equal or reduced dose compared with attenuation-based imaging.

Our group recently tested the feasibility of in-line phase contrast SR micro-CT for imaging the cortical porosity in the forelimb of rats with radiation doses comparable to those commonly employed in vivo for imaging trabecular microarchitecture (78). Since its' first implementation (81), numerous in vivo SR microCT protocols varying in both dose and image resolution have been utilized to visualize the microarchitecture of bone in small animal models $(78,82-84)$. Currently, there is no consensus for what is considered to be a safe dose for live animal in vivo imaging. Voxel sizes in the $10-15 \mu \mathrm{m}$ range are typical for laboratory micro-CT with dose rates of $0.4,0.338$, and $0.939 \mathrm{~Gy}$, respectively (85-87). However, at a voxel size of $11.7 \mu \mathrm{m}$, Matsumoto et al.'s (83) in vivo scans of the knee joint in living mice involving 5 Gy showed no signs of radiation sickness. Based upon this context, our goal was to create an in vivo imaging protocol involving a dose as close as possible to those used routinely in conventional micro-CT and no higher than 5 Gy.

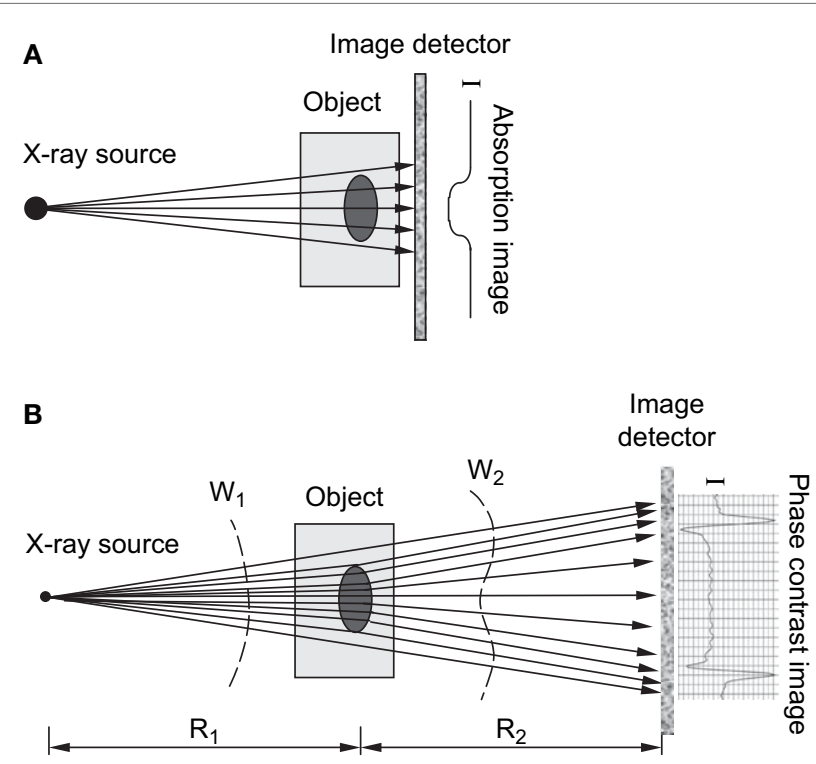

FIGURE 4 | (A) Schematic of attenuation based X-ray imaging where images are produced based on the degree of absorption relative to an object's internal structure. (B) Schematic of in-line phase-contrast imaging based on an object's refractive properties. As X-rays target an object at different angles, variations in the object's internal structure will refract the $X$-rays and cause $a$ shift in the light wave as it propagates through and increasing the object-todetector distance will produce a contrast image (76). Reprint permission granted by the publisher.
In a proof-of-principle study, ex vivo data collected at the biomedical imaging and therapy (BMIT) facility of the Canadian light source (CLS) synchrotron were compared against laboratory micro-CT protocols and their related doses. Studying rat forelimbs, it was found that SR provided superior detection of cortical pores without a substantial increase in dose $(11.8 \mu \mathrm{m}$ voxels, $2.53 \mathrm{~Gy})$ beyond that used in the laboratory systems $(18 \mu \mathrm{m}$ voxels, 1.2-1.5 Gy; $9 \mu \mathrm{m}$ voxels, 11.7-18.2 Gy) (78) (Figure 5). For in-line phase SR micro-CT, the optimal target-to-detector distance for our configuration was found to be $0.9 \mathrm{~m}$. Subsequent to the ex vivo trials, the first in vivo trial was performed (78) and the first longitudinal studies employing this protocol are under way providing encouraging results with respect to tracking individual remodeling events (Figure 6).

Synchrotron radiation micro-CT, taking advantage of in-line phase contrast, has brought about a new opportunity for in vivo imaging to directly test causative hypotheses relating to cortical bone remodeling; however, it too is not without its limitations. Access to synchrotrons is inherently limited due to the relative

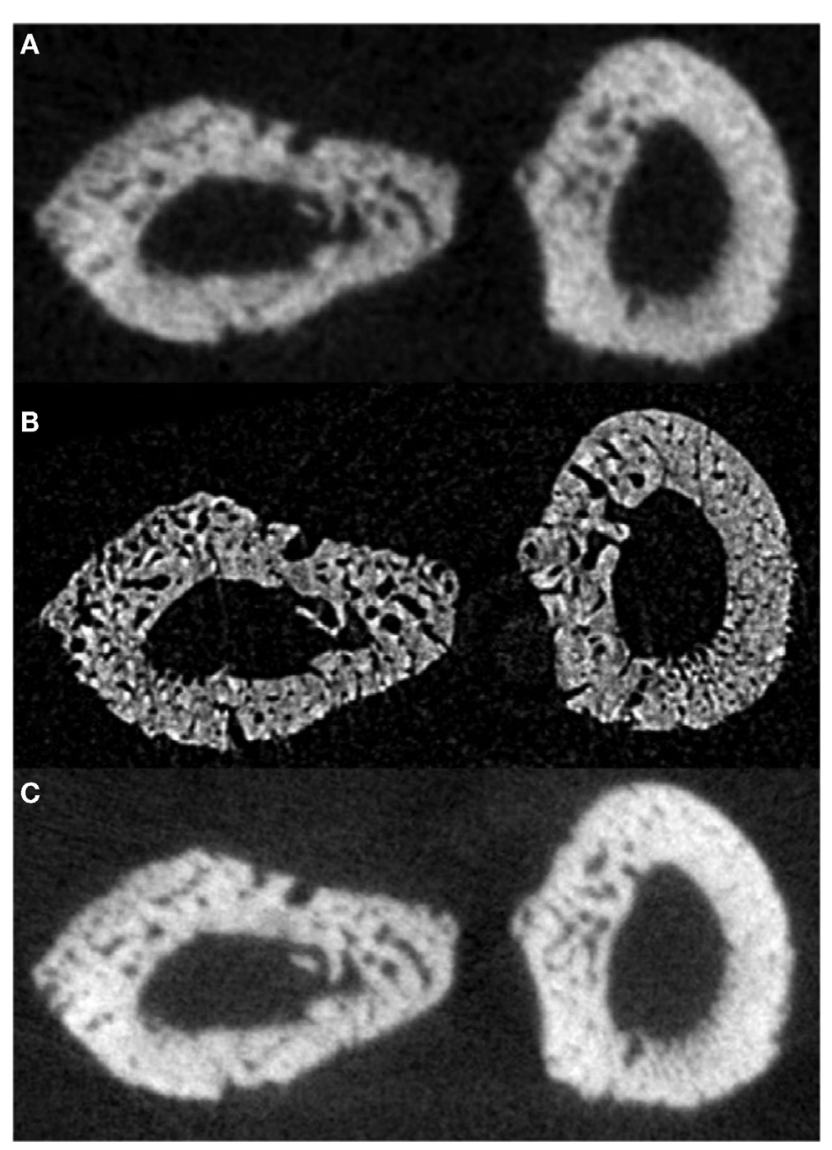

FIGURE 5 | Reconstructed slices of rat forelimbs depicting visualization of cortical porosity based on the imaging system used: (A) in vivo laboratory SkyScan 1176 micro-CT (18 $\mu \mathrm{m}, 1.2-1.5$ Gy dose), (B) in vivo synchrotron micro-CT slice measured using the C4742-56-12HR camera (11.8 $\mu \mathrm{m}, 2.53$ Gy dose), (C) in vivo laboratory SkyScan 1176 (9 $\mu \mathrm{m}, 11.7-18.2$ Gy dose) (78). Reprint permission granted by the publisher. 


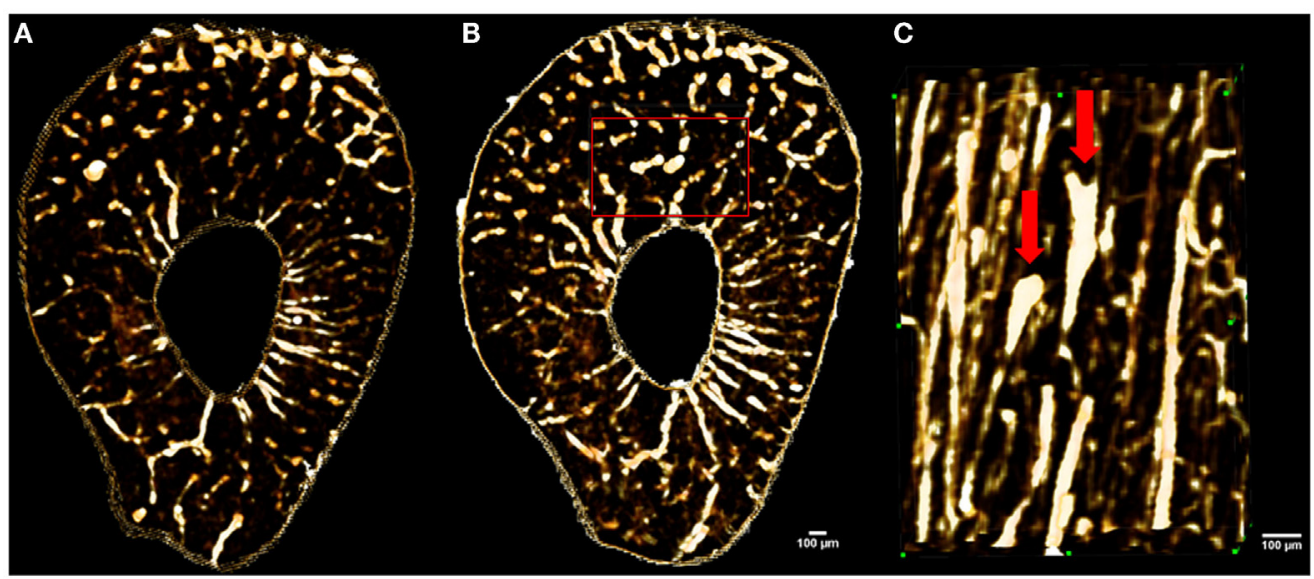

FIGURE 6 | Images showing in vivo matched scans of a rat forelimb acquired with SR micro-CT (11.8 $\mu$, 2.53 Gy). Scan (B) was carried out two weeks after scan (A) on the same rat's forelimb. Image (C) is an enlarged section of image (B) (red rectangle) displaying new remodeling events (red arrows).

scarcity of such facilities and, in particular, those with biomedicalfocused facilities capable of imaging live animals. Even with the high resolution afforded by SR micro-CT and the ability to minimize dose through use of in-line phase contrast imaging, some structures such as osteon borders and microcracks are not observable, in vivo. Indeed, such structures remain challenging to image ex vivo, requiring very high-resolution systems. Movement artifacts are problematic as the difficulty in holding the animal perfectly still increases along with resolution. The smallest of the standard animal models (e.g., mice and rats) have the further drawback of not exhibiting much, if any, natural cortical remodeling - although it can be induced by experimental means [e.g., fatigue loading in the rat (31)]. Notably, this imaging approach has the potential to be extended to larger animal models including those such as the rabbit, which exhibit remodeling under normal conditions. Larger cortical canals in such animals could also relax the resolution needs and, concomitantly, reduce radiation dose.

\section{Conclusion}

The remodeling process, carried out by the activity of BMUs, is of great interest to the bone biology community. This process represents the primary means of skeletal change after maturity and lies at the root of many chronic bone diseases, including osteoporosis. Visualization of cortical bone microarchitecture and, specifically, the remodeling process have progressed from $2 \mathrm{D}$ histological analysis through ex vivo $3 \mathrm{D}$ imaging and now to in vivo $3 \mathrm{D}$ analysis. This progression has paralleled but lagged behind visualization of trabecular bone microarchitecture due to the smaller scale of the target features and the need for high resolution which suffers the complication of increased radiation dose for X-ray based imaging. An important consideration is the caveat that while this progression involves a decreasing level of sample destruction/invasiveness, there is a trade off in terms of the structures one can visualize. The imaging approaches are best suited to detection of porosity. Thus, for some applications, histology (serial or otherwise) remains the most powerful or possibly only approach available. That said, there is a great potential to combine the strengths of these approaches - fusing $2 \mathrm{D}$ and $3 \mathrm{D}$ imaging to maximize the information available. This will enable targeted histology - directed by imaging data. Looking to the near future, we believe that this approach will see application in direct testing of hypotheses related to the regulation of BMU activity - including questions related to steering/orientation, the role of microcracks, and the relation to other stimuli, including possibly cellular signals. Such data will, in turn, prove invaluable for validating in silico models - an area of increasing focus in bone biology. Ultimately, we believe the novel insights possible through 3D data will shed significant new light on the "how" of bone aging, adaptation, and disease. Understanding the "how" is critical to understand the "why."

\section{Acknowledgments}

The authors thank the organizers of this topic, Drs. Phil Salmon, Daniel Chappard, and Andrew Pitsillides. The authors would also like to thank: the Canadian Light Source BMIT line staff, particularly Drs. George Belev, Ning Zhu, and Adam Webb, Dr. Melanie van Der Loop, and staff of the University of Saskatchewan's Laboratory Animal Services Unit, and the support of Dr. Mike Doschak of the Pharmaceutical Orthopedic Research Lab at the University of Alberta, and the contributions of Dr. Dean Chapman, Dr. Yasmin Carter, Isaac Pratt, and Danielle Kabatoff. Finally, the authors thank Ms. Suyoko Tsukamoto for providing black bear bone samples (MB Conservation WBO8992). Support for this research was provided by the Natural Sciences and Engineering Research Council (NSERC) of Canada via a Discovery Grant to DMLC (RGPIN-2014-05563) and the Canadian Foundation for Innovation and Canada Research Chairs program. $\mathrm{KH}$ is supported by the University of Saskatchewan, College of Medicine, and is a CIHR-THRUST Fellow. 


\section{References}

1. Havers C. Osteologia Nova, or, Some New Observations of the Bones and the Parts Belonging to them, with the Manner of their Accretion, and Nutrition, Communicated to the Royal Society in Several Discourses. Ann Arbor, MI: University Microfilms International (1691).

2. Tomes J, Morgan CD. Observations on the structure and development of bone. Philos Trans R Soc Lond B Biol Sci (1853) 143:109-39. doi:10.1098/rstl.1853.0004

3. Wolff J. Das Gesetz der Transformation der Knochen. Berlin: Hirschwald Verlag (1892).

4. Johnson L. Morphologic analysis of pathology. In: Frost H, editor. Bone Biodynamics. Boston, MA: Little, Brown, and Company (1964), 543-54.

5. Rauch F, Travers R, Glorieux FH. Intracortical remodeling during human bone development - a histomorphometric study. Bone (2007) 40:274-80. doi:10.1016/j. bone.2006.09.012

6. Cooper DM, Thomas CD, Clement JG, Turinsky AL, Sensen CW, Hallgrímsson B. Age-dependent change in the 3D structure of cortical porosity at the human femoral midshaft. Bone (2007) 40:957-65. doi:10.1016/j.bone.2006.11.011

7. Rochefort GY. The osteocyte as a therapeutic target in the treatment of osteoporosis. Ther Adv Musculoskelet Dis (2014) 6:79-91. doi:10.1177/1759720X14523500

8. Torres-Del-Pliego E, Vilaplana L, Guerri-Fernandez R, Diez-Perez A. Measuring bone quality. Curr Rheumatol Rep (2013) 15:373. doi:10.1007/s11926-013-0373-8

9. Borah B, Gross GJ, Dufresne TE, Smith TS, Cockman MD, Chmielewski PA, et al. Three-dimensional microimaging (MRmicroI and microCT), finite element modeling, and rapid prototyping provide unique insights into bone architecture in osteoporosis. Anat Rec (2001) 265:101-10. doi:10.1002/ar.1060

10. Frost HM. Tetracycline-based histological analysis of bone remodeling. Calcif Tissue Res (1969) 3:211-37. doi:10.1007/BF02058664

11. Frost HM. The skeletal intermediary organization. Metab Bone Dis Relat Res (1983) 4:281-90. doi:10.1016/S0221-8747(83)80001-0

12. Hert J, Fiala P, Petrtyl M. Osteon orientation of the diaphysis of the long bones in man. Bone (1994) 15:269-77. doi:10.1016/8756-3282(94)90288-7

13. Petrtyl M, Hert J, Fiala P. Spatial organization of the haversian bone in man. $J$ Biomech (1996) 29:161-9. doi:10.1016/0021-9290(94)00035-2

14. Basillais A, Bensamoun S, Chappard C, Brunet-Imbault B, Lemineur G, Ilharreborde $\mathrm{B}$, et al. Three-dimensional characterization of cortical bone microstructure by microcomputed tomography: validation with ultrasonic and microscopic measurements. J Orthop Sci (2007) 12:141-8. doi:10.1007/s00776-006-1104-z

15. Martin RB. Targeted bone remodeling involves BMU steering as well as activation. Bone (2007) 40:1574-80. doi:10.1016/j.bone.2007.02.023

16. Burr DB, Martin RB, Schaffler MB, Radin EL. Bone remodeling in response to in vivo fatigue microdamage. J Biomech (1985) 18:189-200. doi:10.1016/0021-9290(85)90204-0

17. Skedros JG, Sybrowsky CL, Anderson WE, Chow F. Relationships between in vivo microdamage and the remarkable regional material and strain heterogeneity of cortical bone of adult deer, elk, sheep and horse calcanei. J Anat (2011) 219:722-33. doi:10.1111/j.1469-7580.2011.01428.x

18. Skedros JG, Sybrowsky CL, Parry TR, Bloebaum RD. Regional differences in cortical bone organization and microdamage prevalence in Rocky Mountain mule deer. Anat Rec A Discov Mol Cell Evol Biol (2003) 274:837-50. doi:10.1002/ar.a.10102

19. Judex S, Gross TS, Zernicke RF. Strain gradients correlate with sites of exercise-induced bone-forming surfaces in the adult skeleton. J Bone Miner Res (1997) 12:1737-45. doi:10.1359/jbmr.1997.12.10.1737

20. Martin RB, Gibson VA, Stover SM, Gibeling JC, Griffins LV. Osteonal structure in the equine third metacarpus. Bone (1996) 19:165-71. doi:10.1016/8756-3282(96)00167-6

21. Skedros JG, Mendenhall SD, Kiser CJ, Winet H. Interpreting cortical bone adaptation and load history by quantifying osteon morphotypes in circularly polarized light images. Bone (2009) 44:392-403. doi:10.1016/j.bone.2008.10.053

22. Britz HM, Thomas CD, Clement JG, Cooper DM. The relation of femoral osteon geometry to age, sex, height and weight. Bone (2009) 45:77-83. doi:10.1016/j. bone.2009.03.654

23. Smit TH, Burger EH. Is BMU-coupling a strain-regulated phenomenon? A finite element analysis. J Bone Miner Res (2000) 15:301-7. doi:10.1359/ jbmr.2000.15.2.301

24. Burger EH, Klein-Nulend J, Smit TH. Strain-derived canalicular fluid flow regulates osteoclast activity in a remodelling osteon - a proposal. J Biomech (2003) 36:1453-9. doi:10.1016/S0021-9290(03)00126-X
25. van Oers RF, Ruimerman R, Van Rietbergen B, Hilbers PA, Huiskes R. Relating osteon diameter to strain. Bone (2008) 43:476-82. doi:10.1016/j.bone.2008.05.015

26. Martin RB. Is all cortical bone remodeling initiated by microdamage? Bone (2002) 30:8-13. doi:10.1016/S8756-3282(01)00620-2

27. Parfitt AM. Targeted and nontargeted bone remodeling: relationship to basic multicellular unit origination and progression. Bone (2002) 30:5-7. doi:10.1016 S8756-3282(01)00642-1

28. Burr DB, Robling AG, Turner CH. Effects of biomechanical stress on bones in animals. Bone (2002) 30:781-6. doi:10.1016/S8756-3282(02)00707-X

29. Ehrlich PJ, Lanyon LE. Mechanical strain and bone cell function: a review. Osteoporos Int (2002) 13:688-700. doi:10.1007/s001980200095

30. Ryser MD, Nigam N, Komarova SV. Mathematical modeling of spatio-temporal dynamics of a single bone multicellular unit. J Bone Miner Res (2009) 24:860-70. doi:10.1359/jbmr.081229

31. Bentolila V, Boyce TM, Fyhrie DP, Drumb R, Skerry TM, Schaffler MB. Intracortical remodeling in adult rat long bones after fatigue loading. Bone (1998) 23:275-81. doi:10.1016/S8756-3282(98)00104-5

32. Amprino R, Bairati A. Processi di ricostruzione e di riassorbimento nella sostanza compatta delle ossa dell'uomo. Z Zellforsch Mikrosk Anat (1936) 24:439-511. doi:10.1007/BF02462416

33. Parfitt AM, Mathews CH, Villanueva AR, Kleerekoper M, Frame B, Rao DS. Relationships between surface, volume, and thickness of iliac trabecular bone in aging and in osteoporosis. Implications for the microanatomic and cellular mechanisms of bone loss. JClin Invest (1983) 72:1396-409. doi:10.1172/JCI111096

34. Cooper DM, Thomas CD, Clement JG, Hallgrimsson B. Three-dimensional microcomputed tomography imaging of basic multicellular unit-related resorption spaces in human cortical bone. Anat Rec A Discov Mol Cell Evol Biol (2006) 288:806-16. doi:10.1002/ar.a.20344

35. Stout SD, Brunsden BS, Hildebolt CF, Commean PK, Smith KE, Tappen NC. Computer-assisted 3D reconstruction of serial sections of cortical bone to determine the 3D structure of osteons. Calcif Tissue Int (1999) 65:280-4. doi:10.1007/ s002239900699

36. Martin RB. On the histologic measurement of osteonal BMU activation frequency. Bone (1994) 15:547-9. doi:10.1016/8756-3282(94)90279-8

37. Frost HM. Mathematical Elements of Lamellar Bone Remodeling. Springfield, IL: Charles C. Thomas (1964).

38. Frost H. Presence of microscopic cracks in vivo in bone. Henry Ford Hosp Med Bull (1960) 8:35.

39. Lee TC, Mohsin S, Taylor D, Parkesh R, Gunnlaugsson T, O’Brien FJ, et al. Detecting microdamage in bone. J Anat (2003) 203:161-72. doi:10.1046/j.1469-7580.2003.00211.x

40. Presbitero G, O'Brien FJ, Lee TC, Taylor D. Distribution of microcrack lengths in bone in vivo and in vitro. J Theor Biol (2012) 304:164-71. doi:10.1016/j. jtbi.2012.03.027

41. Thurner PJ, Wyss P, Voide R, Stauber M, Stampanoni M, Sennhauser U, et al. Time-lapsed investigation of three-dimensional failure and damage accumulation in trabecular bone using synchrotron light. Bone (2006) 39:289-99. doi:10.1016/j. bone.2006.01.147

42. Larrue A, Rattner A, Peter ZA, Olivier C, Laroche N, Vico L, et al. Synchrotron radiation micro-CT at the micrometer scale for the analysis of the three-dimensional morphology of microcracks in human trabecular bone. PLoS One (2011) 6:e21297. doi:10.1371/journal.pone.0021297

43. Voide R, Schneider P, Stauber M, Wyss P, Stampanoni M, Sennhauser U, et al. Timelapsed assessment of microcrack initiation and propagation in murine cortical bone at submicrometer resolution. Bone (2009) 45:164-73. doi:10.1016/j.bone.2009.04.248

44. Cohen J, Harris WH. The three-dimensional anatomy of haversian systems. $J$ Bone Joint Surg Am (1958) 40:419-34.

45. Tappen NC. Three dimensional studies of resorption spaces and developing osteons. Am J Anat (1977) 149:301-17. doi:10.1002/aja.1001490302

46. Skedros JG, Sorenson SM, Jenson NH. Are distributions of secondary osteon variants useful for interpreting load history in mammalian bones? Cells Tissues Organs (2007) 185:285-307. doi:10.1159/000102176

47. Feldkamp LA, Goldstein SA, Parfitt MA, Jesion G, Kleerekoper M. The direct examination of three-dimensional bone architecture in vitro by computed tomography. J Bone Miner Res (1989) 4:3-11. doi:10.1002/jbmr.5650040103

48. Cooper DM, Turinsky AL, Sensen CW, Hallgrimsson B. Quantitative 3D analysis of the canal network in cortical bone by micro-computed tomography. Anat Rec B New Anat (2003) 274:169-79. doi:10.1002/ar.b.10024 
49. Britz HM, Jokihaara J, Leppanen OV, Jarvinen T, Cooper DM. 3D visualization and quantification of rat cortical bone porosity using a desktop micro-CT system: a case study in the tibia. J Microsc (2010) 240:32-7. doi:10.1111/j.1365-2818.2010.03381.x

50. Pazzaglia UE, Zarattini G, Giacomini D, Rodella L, Menti AM, Feltrin G. Morphometric analysis of the canal system of cortical bone: an experimental study in the rabbit femur carried out with standard histology and micro-CT. Anat Histol Embryol (2010) 39:17-26. doi:10.1111/j.1439-0264.2009.00973.x

51. Chen H, Zhou X, Shoumura S, Emura S, Bunai Y. Age- and gender-dependent changes in three-dimensional microstructure of cortical and trabecular bone at the human femoral neck. Osteoporos Int (2010) 21:627-36. doi:10.1007/ s00198-009-0993-z

52. Palacio-Mancheno PE, Larriera AI, Doty SB, Cardoso L, Fritton SP. 3D assessment of cortical bone porosity and tissue mineral density using high-resolution microCT: effects of resolution and threshold method. J Bone Miner Res (2014) 29:142-50. doi:10.1002/jbmr.2012

53. Bousson V, Peyrin F, Bergot C, Hausard M, Sautet A, Laredo J-D. Cortical bone in the human femoral neck: three-dimensional appearance and porosity using synchrotron radiation. J Bone Miner Res (2004) 19:794-801. doi:10.1359/jbmr.040124

54. Harrison K, Tsukamoto S, Cooper DML. Abstracts - AAPA presentations. Am J Phys Anthropol (2014) 153:64-283.

55. Arhatari BD, Cooper DM, Thomas CD, Clement JG, Peele AG. Imaging the 3D structure of secondary osteons in human cortical bone using phase-retrieval tomography. Phys Med Biol (2011) 56:5265-74. doi:10.1088/0031-9155/56/16/012

56. Dalstra M, Karaj E, Beckmann F, Andersen T, Cattaneo PM. Osteonal mineralization patterns in cortical bone studied by synchrotron-radiation-based computed microtomography and scanning acoustic microscopy. In Bonse $\mathrm{U}$, editor. Developments in X-ray Tomography IV Proc. of SPIE, Vol. 5535. Bellingham: SPIE (2004). p. 143-51.

57. Cooper DM, Erickson B, Peele AG, Hannah K, Thomas CD, Clement JG. Visualization of 3D osteon morphology by synchrotron radiation micro-CT. J Anat (2011) 219:481-9. doi:10.1111/j.1469-7580.2011.01398.x

58. Carter Y, Thomas CD, Clement JG, Peele AG, Hannah K, Cooper DM. Variation in osteocyte lacunar morphology and density in the human femur - a synchrotron radiation micro-CT study. Bone (2013) 52:126-32. doi:10.1016/j.bone.2012.09.010

59. Langer M, Pacureanu A, Suhonen H, Grimal Q, Cloetens P, Peyrin F. X-ray phase nanotomography resolves the 3D human bone ultrastructure. PLoS One (2012) 7:e35691. doi:10.1371/journal.pone.0035691

60. Schneider P, Stauber M, Voide R, Stampanoni M, Donahue LR, Müller R. Ultrastructural properties in cortical bone vary greatly in two inbred strains of mice as assessed by synchrotron light based micro- and nano-CT. J Bone Miner Res (2007) 22:1557-70. doi:10.1359/jbmr.070703

61. Voide R, Schneider P, Stauber M, Van Lenthe GH, Stampanoni M, Muller R. The importance of murine cortical bone microstructure for microcrack initiation and propagation. Bone (2011) 49:1186-93. doi:10.1016/j.bone.2011.08.011

62. Christen D, Levchuk A, Schori S, Schneider P, Boyd SK, Müller R. Deformable image registration and 3D strain mapping for the quantitative assessment of cortical bone microdamage. J Mech Behav Biomed Mater (2012) 8:184-93. doi:10.1016/j.jmbbm.2011.12.009

63. Ford NL, Thornton MM, Holdsworth DW. Fundamental image quality limits for microcomputed tomography in small animals. Med Phys (2003) 30:2869-77. doi:10.1118/1.1617353

64. BritzHM, Carter Y, Jokihaara J, Leppänen OV, Järvinen TL, Belev G, et al. Prolonged unloading in growing rats reduces cortical osteocyte lacunar density and volume in the distal tibia. Bone (2012) 51:913-9. doi:10.1016/j.bone.2012.08.112

65. Mader KS, Schneider P, Muller R, Stampanoni M. A quantitative framework for the 3D characterization of the osteocyte lacunar system. Bone (2013) 57:142-54. doi:10.1016/j.bone.2013.06.026

66. Turnbull TL, Gargac JA, Niebur GL, Roeder RK. Detection of fatigue microdamage in whole rat femora using contrast-enhanced micro-computed tomography. $J$ Biomech (2011) 44:2395-400. doi:10.1016/j.jbiomech.2011.06.032

67. Leng H, Wang X, Ross RD, Niebur GL, Roeder RK. Micro-computed tomography of fatigue microdamage in cortical bone using a barium sulfate contrast agent. $J$ Mech Behav Biomed Mater (2008) 1:68-75. doi:10.1016/j.jmbbm.2007.06.002

68. Landrigan MD, LI J, Turnbull TL, Burr DB, Niebur GL, Roeder RK. Contrastenhanced micro-computed tomography of fatigue microdamage accumulation in human cortical bone. Bone (2011) 48:443-50. doi:10.1016/j.bone.2010.10.160

69. Burghardt AJ, Buie HR, Laib A, Majumdar S, Boyd SK. Reproducibility of direct quantitative measures of cortical bone microarchitecture of the distal radius and tibia by HR-pQCT. Bone (2010) 47:519-28. doi:10.1016/j.bone.2010.05.034
70. Jorgenson BL, Buie HR, Mcerlain DD, Sandino C, Boyd SK. A comparison of methods for in vivo assessment of cortical porosity in the human appendicular skeleton. Bone (2015) 73:167-75.

71. MacDonald HM, Nishiyama KK, Hanley DA, Boyd SK. Changes in trabecular and cortical bone microarchitecture at peripheral sites associated with 18 months of teriparatide therapy in postmenopausal women with osteoporosis. Osteoporosis International (2011) 22:357-62.

72. Engelke K, Libanati C, Fuerst T, Zysset P, Genant HK. Advanced CT based in vivo methods for the assessment of bone density, structure, and strength. Curr Osteoporos Rep (2013) 11:246-55. doi:10.1007/s11914-013-0147-2

73. Pacheco R, Stock H. Effects of radiation on bone. Curr Osteoporos Rep (2013) 11:299-304. doi:10.1007/s11914-013-0174-z

74. Laperre K, Depypere M, van Gastel N, Torrekens S, Moermans K, Bogaerts $\mathrm{R}$, et al. Development of micro-CT protocols for in vivo follow-up of mouse bone architecture without major radiation side effects. Bone (2011) 49:613-22. doi:10.1016/j.bone.2011.06.031

75. Klinck RJ, Campbell GM, Boyd SK. Radiation effects on bone architecture in mice and rats resulting from in vivo micro-computed tomography scanning. Med Eng Phys (2008) 30:888-95. doi:10.1016/j.medengphy.2007.11.004

76. Zhou S-A, Brahme A. Development of phase-contrast X-ray imaging techniques and potential medical applications. Phys Med (2008) 24:129-48. doi:10.1016/j. ejmp.2008.05.006

77. Arfelli F, Assante M, Bonvicini V, Bravin A, Cantatore G, Castelli E, et al. Low-dose phase contrast x-ray medical imaging. Phys Med Biol (1998) 43:2845. doi:10.1088/0031-9155/43/10/013

78. Pratt IV, Belev G, Zhu N, Chapman LD, Cooper DM. In vivo imaging of rat cortical bone porosity by synchrotron phase contrast micro computed tomography. Phys Med Biol (2015) 60:211-32. doi:10.1088/0031-9155/60/1/211

79. Carter Y, Thomas CD, Clement JG, Cooper DM. Femoral osteocyte lacunar density, volume and morphology in women across the lifespan. J Struct Biol (2013) 183:519-26. doi:10.1016/j.jsb.2013.07.004

80. Carter Y, Suchorab JL, Thomas CD, Clement JG, Cooper DM. Normal variation in cortical osteocyte lacunar parameters in healthy young males. J Anat (2014) 225:328-36. doi:10.1111/joa.12213

81. Kinney JH, Lane NE, Haupt DL. In vivo, three-dimensional microscopy of trabecular bone. J Bone Miner Res (1995) 10:264-70. doi:10.1002/jbmr.5650100213

82. Bayat S, Apostol L, Boller E, Brochard T, Peyrin F. In vivo imaging of bone micro-architecture in mice with 3D synchrotron radiation micro-tomography. Nucl Instrum Methods Phys Res A (2005) 548:247-52. doi:10.1016/j.nima.2005.03.097

83. Matsumoto T, Nishikawa K, Tanaka M, Uesugi K. In vivo CT quantification of trabecular bone dynamics in mice after sciatic neurectomy using monochromatic synchrotron radiation. Calcif Tissue Int (2011) 88:432-41. doi:10.1007/ s00223-011-9475-3

84. Coan P, Wagner A, Bravin A, Diemoz PC, Keyriläinen J, Mollenhauer J. In vivo $\mathrm{X}$-ray phase contrast analyzer-based imaging for longitudinal osteoarthritis studies in guinea pigs. Phys Med Biol (2010) 55:7649. doi:10.1088/0031-9155/55/24/017

85. Waarsing JH, Day JS, Van der Linden JC, Ederveen AG, Spanjers C, De Clerck N, et al. Detecting and tracking local changes in the tibiae of individual rats: a novel method to analyse longitudinal in vivo micro-CT data. Bone (2004) 34:163-9. doi:10.1016/j.bone.2003.08.012

86. Campbell G, Ominsky M, Boyd S. Bone quality is partially recovered after the discontinuation of RANKL administration in rats by increased bone mass on existing trabeculae: an in vivo micro-CT study. Osteoporos Int (2011) 22:931-42. doi:10.1007/s00198-010-1283-5

87. Brouwers JE, van Rietbergen B, Huiskes R. No effects of in vivo micro-CT radiation on structural parameters and bone marrow cells in proximal tibia of wistar rats detected after eight weekly scans. J Orthop Res (2007) 25:1325-32. doi:10.1002/ jor.20439

Conflict of Interest Statement: The authors declare that the research was conducted in the absence of any commercial or financial relationships that could be construed as a potential conflict of interest.

Copyright $(2) 2015$ Harrison and Cooper. This is an open-access article distributed under the terms of the Creative Commons Attribution License (CC BY). The use, distribution or reproduction in other forums is permitted, provided the original author(s) or licensor are credited and that the original publication in this journal is cited, in accordance with accepted academic practice. No use, distribution or reproduction is permitted which does not comply with these terms. 